\title{
Evaluation Des Teneurs En Eléments Traces Métalliques (Cadmium Et Plomb) Dans L’eau, Les Sédiments Et Deux Espèces De Poissons Clarias gariepinus (Burchell, 1822) Et Oreochromis niloticus (Linné, 1758) Dans Le Pool Malebo (Fleuve Congo), RD Congo
}

\author{
Jeff Nakweti K., BSc \\ Département de Biologie, Faculté des Sciences, Université de Kinshasa \\ (UNIKIN), Kinshasa XI, R.D Congo \\ Willy Lusasi Swana, BSc \\ John Tembeni M., PhD \\ Laboratoire de Limnologie, Hydrobiologie et Aquaculture, Département de \\ Biologie, Faculté des Sciences, Université de Kinshasa (UNIKIN), Kinshasa

$$
\text { XI, R.D Congo }
$$

\section{Doi:10.19044/esj.2021.v17n25p174}

Submitted: 23 February 2021

Accepted: 26 April 2021

Published: 31 July 2021
Copyright 2021 Author(s)

Under Creative Commons BY-NC-ND 4.0 OPEN ACCESS

Cite As:

Nakweti K.J., Lusasi S.W. \& Tembeni M.J. (2021). Evaluation Des Teneurs En Eléments Traces Métalliques (Cadmium Et Plomb) Dans L'eau, Les Sédiments Et Deux Espèces De Poissons Clarias Gariepinus (Burchell, 1822) Et Oreochromis Niloticus (Linné, 1758) Dans Le Pool Malebo (Fleuve Congo), RD Congo. European Scientific Journal, ESJ, 17(25), 174. https://doi.org/10.19044/esj.2021.v17n25p174

\section{Résumé}

Cette étude a pour objectif d'évaluer les niveaux de contamination en Eléments Traces Métalliques (Cadmium et Plomb) dans l'eau, les sédiments et dans l'organisme de deux espèces de poissons, pélagique (Oreochromis niloticus Linné, 1758) et benthique (Clarias gariepinus Burchell, 1822). Elle a eu lieu de Juillet 2019 à Octobre 2019, dans le Pool Malebo (fleuve Congo/Kinshasa) à Kingabwa, sur deux sites (Baramoto et Mongole). Les analyses des Eléments Traces Métalliques ont été effectuées à l'aide du spectrophotomètre UV-visible. Les teneurs en ETM dans les eaux ont été respectivement de $0,01 \pm 0,001$ pour le $\mathrm{Cd}(\mathrm{mg} / \mathrm{L})$ et $0,05 \pm 0,03$ pour le $\mathrm{Pb}$ $(\mathrm{mg} / \mathrm{L})$ à Baramoto, et de 0,02 $\pm 0,01$ pour le Cd (mg/L), et 0,01 $\pm 0,005$ pour le 
$\mathrm{Pb}(\mathrm{mg} / \mathrm{L})$ à Mongole. Ces teneurs sont supérieures aux seuils recommandés par l’OMS (Cd: 0,003 mg/L ; Pb: 0,01 mg/L). L'analyse des sédiments des deux sites a révélé des teneurs généralement faibles, en dessous des seuils recommandés par le Conseil Canadien des Ministres de l'Environnement (CCME). Par ailleurs, les teneurs de ces deux ETM ont montré une certaine variabilité relative dans les branchies et les muscles de deux espèces étudiées. Ces teneurs dépassent les seuils de recommandation de l'OMS (Cd: 0,05 $\mathrm{mg} / \mathrm{kg}$; $\mathrm{Pb}: 0,2 \mathrm{mg} / \mathrm{kg}$ ) sur la comestibilité des poissons. L'espèce pélagique, Oreochromis niloticus (Cd: 0,179 $\pm 0,086 \mathrm{mg} / \mathrm{kg} ; \mathrm{Pb}: 0,249 \pm 0,117 \mathrm{mg} / \mathrm{kg}$ ) a montré des teneurs élevées que l'espèce benthique, Clarias gariepinus (Cd: 0,173 $\pm 0,097 \mathrm{mg} / \mathrm{kg}$; Pb: 0,219 $\pm 0,096 \mathrm{mg} / \mathrm{kg}$ ). Des plus, chez les deux espèces, Les branchies $(\mathrm{Pb}: 0,272 \pm 0,075 \mathrm{mg} / \mathrm{kg}$ à $0,263 \pm 0,055 \mathrm{mg} / \mathrm{kg} ; \mathrm{Cd}$ : $0,219 \pm 0,122 \mathrm{mg} / \mathrm{kg}$ à $0,208 \pm 0,055 \mathrm{mg} / \mathrm{kg}$ ) concentrent plus d'Eléments Traces que les muscles (Pb: 0,166 $\pm 0,081 \mathrm{mg} / \mathrm{kg}$ à $0,236 \pm 0,082 \mathrm{mg} / \mathrm{kg}$; Cd: $0,127 \pm 0,085 \mathrm{mg} / \mathrm{kg}$ à $0,150 \pm 0,063 \mathrm{mg} / \mathrm{kg}$ ). La consommation régulière de ces poissons pourrait engendrée des effets néfastes à la santé humaine.

Mots clés : Pool Malebo, Fleuve Congo, Eléments Traces Métalliques, Poissons, Sédiment

\title{
Evaluation Of Trace Metal Elements (Cadmium And Lead) In Water, Sediment And Two Fish Species Clarias gariepinus (Burchell, 1822) And Oreochromis niloticus (Linnaeus, 1758) In Malebo Pool (Congo River), DR. Congo
}

\author{
Jeff Nakweti K., BSc \\ Department of Biology, Faculty of Sciences, University of Kinshasa \\ (UNIKIN),Kinshasa XI, Democratic Republic of Congo \\ Willy Lusasi Swana, BSc \\ John Tembeni M., PhD \\ Laboratory of Limnology, Hydrobiology and Aquaculture, Department of \\ Biology, Faculty of Sciences, University of Kinshasa (UNIKIN), Kinshasa \\ XI, Democratic Republic of Congo
}

\begin{abstract}
The objective of this study is to assess the levels of contamination of Metal Trace Elements (Cadmium and Lead) in the water, sediments and in the organism of two species of fish, pelagic (Oreochromis niloticus Linnaeus, 1758) and benthic (Clarias gariepinus Burchell, 1822). It took place from July 2019 to October 2019, in the Pool Malebo (Congo River / Kinshasa) in Kingabwa, on two sites (Baramoto and Mongole). The analyzes of the Metal
\end{abstract}


Trace Elements were carried out using the UV-visible spectrophotometer. The ETM contents in the water were respectively $0.01 \pm 0.001$ for Cd $(\mathrm{mg} / \mathrm{L})$ and $0.05 \pm 0.03$ for $\mathrm{Pb}(\mathrm{mg} / \mathrm{L})$ at Baramoto, and $0.02 \pm 0,01$ for $\mathrm{Cd}(\mathrm{mg} / \mathrm{L})$, and $0.01 \pm 0.005$ for $\mathrm{Pb}(\mathrm{mg} / \mathrm{L})$ in Mongolian. These levels are above the thresholds recommended by WHO (Cd: $0.003 \mathrm{mg} / \mathrm{L}$; Pb: $0.01 \mathrm{mg} / \mathrm{L}$ ). Analysis of the sediments from both sites revealed generally low levels, below the thresholds recommended by the Canadian Council of Ministers of the Environment (CCME). In addition, the contents of these two ETM showed a certain relative variability in the gills and the muscles of two species studied. These levels exceed the WHO recommendation thresholds (Cd: $0.05 \mathrm{mg} / \mathrm{kg}$; $\mathrm{Pb}: 0.2 \mathrm{mg} / \mathrm{kg}$ ) on the edibility of fish. The pelagic species, Oreochromis niloticus (Cd: $0.179 \pm 0.086 \mathrm{mg} / \mathrm{kg}$; Pb: $0.249 \pm 0.117 \mathrm{mg} / \mathrm{kg}$ ) showed higher levels than the benthic species, Clarias gariepinus (Cd: $0.173 \pm 0.097$ $\mathrm{mg} / \mathrm{kg} ; \mathrm{Pb}: 0.219 \pm 0.096 \mathrm{mg} / \mathrm{kg}$ ). In addition, in both species, the gills (Pb: $0.272 \pm 0.075 \mathrm{mg} / \mathrm{kg}$ to $0.263 \pm 0.055 \mathrm{mg} / \mathrm{kg}$; Cd: $0.219 \pm 0.122 \mathrm{mg} / \mathrm{kg}$ to $0.208 \pm 0.055 \mathrm{mg} / \mathrm{kg}$ ) concentrate more Trace Elements than muscles (Pb: $0.166 \pm 0.081 \mathrm{mg} / \mathrm{kg}$ to $0.236 \pm 0.082 \mathrm{mg} / \mathrm{kg}$; Cd: $0.127 \pm 0.085 \mathrm{mg} / \mathrm{kg}$ to $0.150 \pm 0.063 \mathrm{mg} / \mathrm{kg})$. Regular consumption of these fish could have harmful effects on human health.

Keywords: Malebo Pool, Congo River, Trace Metal Elements, Fish, Sediment

\section{Introduction}

Depuis quelques années, la consommation des ressources halieutiques, spécifiquement les poissons, se trouve au centre de l'actualité par rapport aux éléments bénéfiques qu'elles procurent à la santé humaine, tels que les acides gras essentiels, les protéines, les vitamines $\mathrm{A}, \mathrm{D}$ et $\mathrm{E}$ ainsi que les éléments minéraux. Cependant, ils sont également exposés à certains contaminants toxiques de l'environnement tels que les Eléments Traces Métalliques (ETM: le cadmium, le plomb, etc.) qui s'accumulent dans leurs tissus et se transfèrent dans la chaine alimentaire jusqu'à l'homme (Chadid, 2016).

La problématique du transfert des contaminants métalliques de l'environnement vers la chaine alimentaire est une source d'inquiétudes et un sujet de nombreuses recherches à travers le monde (Falasi, 2018). Elle a attiré l'attention des plusieurs chercheurs depuis que l'industrialisation a apporté une augmentation des contaminants dans les écosystèmes aquatiques qui, sont utilisés comme décharges par les humains. Il en résulte des perturbations de l'équilibre écologique. Ce problème a tout d'abord était mis en évidence dans les pays industrialisés (Ouedraogo, 2016).

Bien que l'activité industrielle soit moins développée dans la plupart des pays Africains, une prise de conscience croissante a été observée à travers des nombreuses études quant à la nécessité de gérer rationnellement les ressources 
aquatiques et de maîtriser les déversements des déchets dans l'environnement (Ouedraogo, 2016).

La République Démocratique du Congo, bien qu'ayant un réseau industriel très peu développé, n’est pas épargnée par la pollution métallique. Quelques études ont été entreprises dans le cadre de l'évaluation de l'impact des concentrations en ETM dans certains écosystèmes aquatiques (Monama et al., 1985 ; Foxall et al., 2000 ; Katemo et al., 2010 ; Muteba et al., 2011 ; Nsimanda et al., 2015a et b ; Kashimbo et al., 2016 ; Nzapo et al., 2018). Ces travaux ont mis en évidence la présence des Eléments Traces Métalliques dans la chaine trophique.

Dans la ville province de Kinshasa, le Pool Malebo n'échappe pas à ce constat. Avec le développement des activités urbaines, ce plan d'eau est devenu le réceptacle de rejets anthropiques (industrielles, agricoles, domestiques, etc..) ou d'origines naturelles chargés en éléments métalliques toxiques $(\mathrm{Pb}, \mathrm{Cd}, \mathrm{Hg}$, etc.) qui se concentrent dans différents compartiments environnementaux.

Des études antérieures portées sur la bioaccumulation des Eléments Traces Métalliques chez les espèces de poissons ainsi que les risques sanitaires liés à leur consommation (Nsimanda et al., 2015a et b ; Nzapo et al., 2018) et sur le degré de contamination en ETM dans les sédiments du Pool Malebo (Mwanamoki et al., 2014; Mata et al., 2020) ont mis en évidence l'existence d'une contamination métallique dans le Pool Malebo. Cependant, la détermination du niveau de cette contamination dans l'eau, les sédiments et les poissons suivant leur habitat n’a jusqu'alors été développé.

Dans ce contexte, nous nous sommes engagés d'évaluer la teneur en Eléments Traces Métalliques ( $\mathrm{Cd}$ et $\mathrm{Pb}$ ) dans l'eau, les sédiments ainsi que dans les tissus de deux espèces de poissons, benthique (Clarias gariepinus Burchell, 1822) et pélagique (Oreochromis niloticus Linné, 1758) dans le Pool Malebo (fleuve Congo).

\section{Matériel et méthodes}

\section{Milieu d'étude}

Cette étude a eu lieu dans le Pool Malebo plus spécifiquement aux sites de Baramoto et Mongole situés à la station de pêche de Kingabwa dans la commune de Limete à Kinshasa en République Démocratique du Congo. La station de Kingabwa a été choisie en raison de sa vocation industrielle, de l'activité de pêche, en plus, du fait qu'elle est soumise à des sources de pollution d'origine urbaine et agricole (Falasi, 2018).

Le Pool Malebo est un lac formé par le fleuve Congo et ses cours moyen, alimenter par d'importants cours d'eau permanents (les rivières: N'djili, Kalamu, N'sele et Bitshaku-tshaku). Il est formé d'un élargissement du fleuve Congo à $35 \mathrm{~km}$ de long et de $25 \mathrm{~km}$ de large. Sa superficie est de $500 \mathrm{~km}^{2}$ et 
il est situé entre la R.D.C (ville province de Kinshasa) et la République du Congo (préfecture de Brazzaville). Le Pool est situé à $4^{\circ} 5^{\prime}$ 'à $4^{\circ} 20^{\prime}$ 'Sud et de $15^{\circ} 19^{\prime}$ à $15^{\circ} 30^{\prime}$ Est et à une altitude de $275 \mathrm{~m}$. Il occupe le fond d'une cuvette entourée de collines dépassant souvent $500 \mathrm{~m}$ (Pwema et al., 2019). La partie centrale du Pool Malebo est occupée par l'île M’bamou en République du Congo et par plusieurs groupes de petits îles dont un archipel se trouve à l'embouchure de la rivière N'djili. D'après la classification de Koppen, le Pool Malebo connaît un climat tropical du type $\mathrm{AW}_{4}$, c'est-à-dire climat tropical humide (A) caractérisé par une période sèche (W) de 4 mois. Ayant une pluviosité moyenne annuelle de $1400 \mathrm{~mm}$, la température moyenne annuelle de l'eau est de $24,6^{\circ} \mathrm{C}$ (Nzapo et al., 2018).

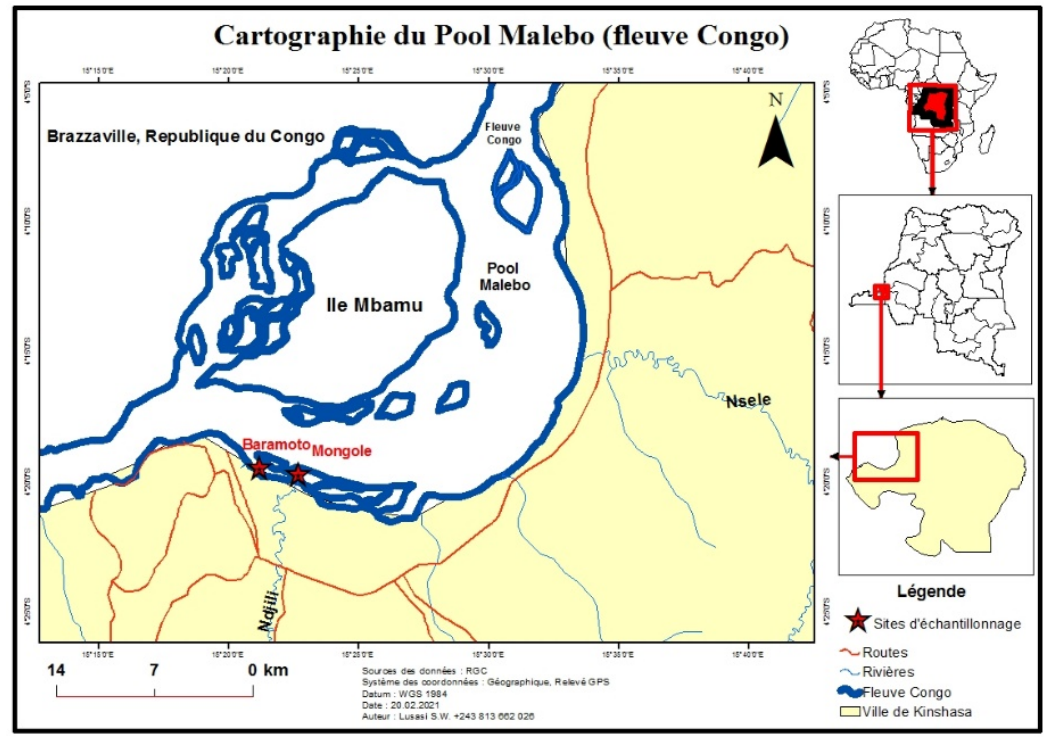

Figure 1. Cartographie du Pool Malebo (fleuve Congo) reprenant les sites d'échantillonnage

\section{Matériel biologique}

Deux espèces des poissons ont été utilisées comme matériel biologique en fonction de leur habitat, leur abondance et leur importance économique. Il s'agit de Clarias gariepinus et Oreochromis niloticus (figures $2 \& 3$ ).
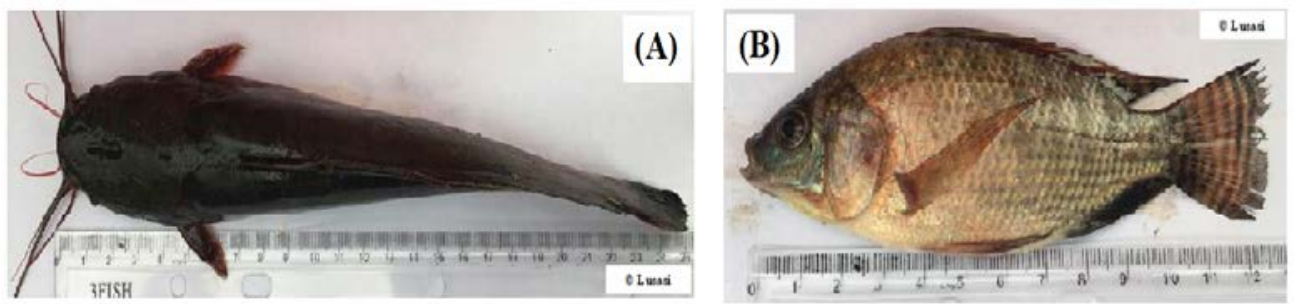

Figures 2 \& 3. Clarias gariepinus (A) \& Oreochromis niloticus (B) (Photos Nakweti, Lusasi \& Tembeni, 2020) 


\section{Méthodes}

\section{Echantillonnage}

Le prélèvement d'échantillons s'est déroulé sur une période allant de Juillet 2019 à Octobre 2019, sur deux sites d'études (Baramoto et Mongole). Les poissons ont été capturés par la technique de pêche artisanale, à l'aide d'un filet épervier de $2 \mathrm{~cm}$ de maille et de $5 \mathrm{~m}$ de diamètre, et d'un filet maillant longs de 24 à $30 \mathrm{~cm}$ sur 1 à $1,5 \mathrm{~m}$ de largeur. Au total 80 individus de poissons ont été échantillonnés dont 35 spécimens de Oreochromis niloticus et 45 de Clarias gariepinus, mis dans des sachets en polyéthylène étiquetés et placés sous glacière bien fermé.

Les spécimens capturés ont été acheminés au Laboratoire de Limnologie, Hydrobiologie et Aquaculture de la Faculté des Sciences de l'Université de Kinshasa et conservés au froid à $-4{ }^{\circ} \mathrm{C}$ jusqu'à l'identification systématique et les analyses toxicologiques ultérieures.

Sur chaque site, seuls les sédiments de surface ont fait l'objet de prélèvements. Au moins $200 \mathrm{~g}$ de sédiment meuble ont été collectés à l'aide d'une perche, conditionnés dans des sachets en polyéthylène numérotés, indiquant le site de prélèvement. L'eau a été prélevée à l'aide des bouteilles en plastique de 1,5 L numérotés. Ces prélèvements ont été conservés dans une glaciaire ensuite transporter au Laboratoire de Géochimie du Centre de Recherche Géologique et Minière de Kinshasa où ils ont été d'abord placés au froid $\left(-4{ }^{\circ} \mathrm{C}\right)$ avant les analyses. Au cours de nos campagnes d'échantillonnage, la température, la conductivité et le $\mathrm{pH}$ de l'eau ont été mesurés in situ à l'aide d'une sonde multi paramétrique Combo (HANNA).

\section{Identification des poissons et préparation d'échantillons}

Les spécimens ont été identifiés à l'aide de clés d'identification systématique proposées par Lévêque et al., (1990 et 1992); Poll et Gosse (1995); Mbenga et Teugels (2003), ensuite classés en lots de tailles en fonction de leur longueur totale (Lt). Les lots ont été définis sur la base de la règle de Sturge selon la formule suivante:

$$
\mathrm{NC}=1+(3.3 \log \mathrm{N})
$$

Où NC: nombre de classe; $\mathrm{N}$ : nombre total d'individus pour l'échantillon considéré. L’intervalle entre les classes (IC) a été déterminé par le rapport suivant :

$$
\text { IC }=\frac{\text { Taille maximale }- \text { taille minimale }}{\text { Nombre total de classes }}
$$

Cinq gammes de taille ont été constituées. Les individus ont ensuite été répartis en deux groupes selon leur stade de croissance (adulte et juvénile) suivant Leprieur et Rubin (2011). Chez Oréochromis niloticus (adulte: $\mathrm{Lt} \geq 14$ cm; juvénile: Lt $<14 \mathrm{~cm}$ ); pour Clarias gariepinus (adulte: Lt $\geq 30,8 \mathrm{~cm}$; 
juvénile: Lt $<$ 30,8 cm). Pour chaque groupe constitué, 3 spécimens ont été tirés aux hasards pour les analyses.

Les analyses toxicologiques ont été effectuées au Laboratoire de Géochimie du Centre de Recherches Géologiques et Minières de Kinshasa. La première opération a consisté d'abord à la dissection des poissons afin de prélever les branchies et les muscles. Les organes ont été séchés à l'étuve (DRY Line) à $105^{\circ} \mathrm{C}$ pendant 48 heures afin d'obtenir la MS (Matière Sèche). Ces MS ont été broyées dans le mortier en porcelaine avec pilon. $2 \mathrm{~g}$ de MS était introduit dans un creuset en porcelaine et calciner pendant $2 \mathrm{~h}$ au four à moufle à $450{ }^{\circ} \mathrm{C}$ jusqu'à l'obtention des cendres. Ces cendres obtenues ont été refroidit et conservées dans un dessiccateur jusqu'à la minéralisation. 1g des cendres de chaque échantillon a été attaqué avec $30 \mathrm{ml}$ de $\mathrm{HCl}$ (10\%) dans un bécher de $100 \mathrm{ml}$. La solution obtenue a été filtrée sur du papier filtre sans cendre, puis ramener à $100 \mathrm{ml}$ avec de l'eau distillée.

Les sédiments humides ont été d'abord séchés à l'étuve à $105{ }^{\circ} \mathrm{C}$ pendant 48h, puis broyés et soumis à l'attaque acide avec $30 \mathrm{ml}$ de $\mathrm{HCl}$ (10\%). Le minéralisât obtenu a été filtré à l'aide du papier filtre dans une fiole de 100 ml. Le volume était ajusté à $100 \mathrm{ml}$ avec de l'eau distillée.

Les échantillons d'eau ont été décongelés à la température ambiante du laboratoire, ensuite filtrés dans un ballon jaugé de $100 \mathrm{ml}$ à l'aide du papier filtre. Le filtrat obtenu a été soumis au dosage spectrophotométrique.

\section{Méthode d'analyse des échantillons}

Les dosages d'ETM, ont été réalisés par la spectrophotométrie UVvisible à l'aide du Spectrophomètre DR-4000U. Nous avons prélevés $25 \mathrm{ml}$ dans la solution à $100 \mathrm{ml}$ obtenue, les échantillons ont été colorés par une réaction de complexation en utilisant la dithizone pour le Cd et une solution d'acide phosphorique pour le $\mathrm{Pb}$. Le produit coloré obtenu est déposé dans l'analyseur. La lecture sur spectrophotomètre a été effectuée suivant une longueur d'onde spécifique. Les longueurs d'ondes ont été fixées à $540 \mathrm{~nm}$ pour le $\mathrm{Cd}$ et $283 \mathrm{~nm}$ pour le $\mathrm{Pb}$. Les concentrations obtenues ont été déterminés en se rapportant à une gamme d'étalonnage.

\section{Facteur de Bioconcentration (FBC)}

Les Facteurs de Bioconcentration ont été calculés par la formule utilisée par Casas (2005), à partir des données des analyses de l'échantillon d'eau de chaque site et celles des poissons:

$$
\mathrm{FBC}=\frac{C o}{C e}
$$

Où, Co: concentration en ETM dans l'organisme; Ce: concentration environnementale (eau). Si le FBC est supérieur à 1 , il y a une bioconcentration de l'Elément Trace. 


\section{Analyse et traitement statistique des données}

Le logiciel Microsoft Excel 2010 a été utilisé pour le traitement des données. Le test de Student a été appliqué pour comparer les moyennes des teneurs en ETM entre les groupes de poissons étudiés. Le logiciel Origin 6.1 a été utilisé pour cette fin. Les différences ont été considérées comme significatives à des valeurs $\mathrm{p}<0,05$. Les résultats obtenus ont été présentés sous formes des tableaux.

\section{Résultats}

\section{Paramètres physico-chimiques de l'eau}

Les valeurs moyennes des paramètres physico-chimiques des eaux enregistrées sur les sites de Baramoto et Mongole sont reprises dans le tableau 1.

Tableau 1. Valeurs moyennes des paramètres physico-chimiques mesurées sur les deux sites de prélèvement de notre étude

\begin{tabular}{|c|c|c|c|}
\hline \multirow{2}{*}{ Paramètres } & \multicolumn{3}{|c|}{ Sites d'échantillonnage } \\
\cline { 2 - 4 } & Baramoto & Mongole & Normes OMS (2011) \\
\hline $\mathrm{pH}$ & $6,51 \pm 0,42$ & $6,64 \pm 0,78$ & $6,5-8,5$ \\
\hline Conductivité $(\mu \mathrm{S} / \mathrm{cm})$ & $29,3 \pm 2,2$ & $27,8 \pm 2,7$ & $50-2000$ \\
\hline Température $\left({ }^{\circ} \mathrm{C}\right)$ & $26,4 \pm 1,27$ & $27,3 \pm 0,79$ & $25-29$ \\
\hline
\end{tabular}

Les résultats d'analyse de l'eau montrent que, le $\mathrm{pH}$ des eaux de deux sites est légèrement acide, soit 6,51 $\pm 0,42$ à Baramoto et $6,64 \pm 0,78$ à Mongole. La conductivité de l'eau des deux sites varie entre 29,3 $\pm 2,2 \mu \mathrm{S} / \mathrm{cm}$ à Baramoto et 27,8 $\pm 2,7 \mu \mathrm{S} / \mathrm{cm}$ à Mongole. Ceci traduit une faible minéralisation des eaux au niveau des sites étudiés. La température de l'eau de surface, sur l'ensemble des sites varie entre $26,4 \pm 1,27^{\circ} \mathrm{C}$ à Baramoto et $27,3 \pm 0,79{ }^{\circ} \mathrm{C}$ à Mongole.

\section{Teneurs en Eléments Traces Métalliques dans les eaux et les sédiments}

Les résultats des teneurs en Eléments Traces Métalliques obtenus dans les eaux et les sédiments des sites de Baramoto et Mongole sont consignés dans le tableau 2.

Tableau 2. Teneurs en ETM des eaux et sédiments mesurées au niveau des deux sites d'étude

\begin{tabular}{|c|c|c|c|c|}
\hline \multirow{2}{*}{ Sites } & \multicolumn{2}{|c|}{ Eaux } & \multicolumn{2}{c|}{ Sédiments } \\
\cline { 2 - 5 } & $\mathbf{C d}(\mathbf{m g} / \mathbf{L})$ & $\mathbf{P b}(\mathbf{m g} / \mathbf{L})$ & $\mathbf{C d}(\boldsymbol{\mu g} / \mathbf{g})$ & $\mathbf{P b}(\boldsymbol{\mu g} / \mathbf{g})$ \\
\hline Baramoto & $0,01 \pm 0,001$ & $0,05 \pm 0,03$ & $0,04 \pm 0,02$ & $0,02 \pm 0,01$ \\
\hline Mongole & $0,02 \pm 0,01$ & $0,01 \pm 0,005$ & $0,01 \pm 0,00$ & $0,01 \pm 0,006$ \\
\hline Normes OMS (2017) & 0,003 & 0,01 & - & - \\
\hline Normes CCME & - & - & 600 & 35000 \\
\hline
\end{tabular}

L'analyse du tableau 2 indique que les teneurs moyennes en $\mathrm{Pb}$ varient entre $0,01 \pm 0,005 \mathrm{mg} / \mathrm{L}$ et $0,05 \pm 0,03 \mathrm{mg} / \mathrm{L}$ respectivement dans les eaux de 
Mongole et de Baramoto. Pour le Cd, les teneurs moyennes varient entre

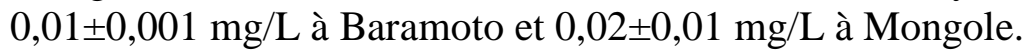

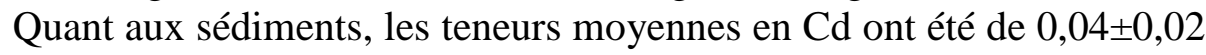
$\mu \mathrm{g} / \mathrm{g}$ et de $0,01 \pm 0,00 \mu \mathrm{g} / \mathrm{g}$ respectivement à Baramoto et à Mongole; par contre, celles de $\mathrm{Pb}$ ont varié entre $0,01 \pm 0,006 \mu \mathrm{g} / \mathrm{g}$ (à Mongole) et 0,02 $\pm 0,01$ $\mu \mathrm{g} / \mathrm{g}$ (à Baramoto).

\section{Teneurs en ETM des poissons de la station de Kingabwa}

Les teneurs moyennes en Eléments Traces Métalliques (Pb et $\mathrm{Cd}$ ) chez les deux espèces de poissons étudiées dans la station de pêche de Kingabwa sont présentées dans le tableau 3.

Tableau 1. Teneurs moyennes en ETM de deux espèces des poissons capturées à la station de pêche de Kingabw

\begin{tabular}{|c|c|c|}
\hline \multirow{2}{*}{ Espèces } & \multicolumn{2}{|c|}{ Eléments Traces Métalliques } \\
\cline { 2 - 3 } & $\mathbf{P b} \mathbf{( m g} / \mathbf{k g})$ & $\mathbf{C d ~} \mathbf{( m g} / \mathbf{k g})$ \\
\hline C. gariepinus & $0,219 \pm 0,096$ & $0,173 \pm 0,097$ \\
\hline O. niloticus & $0,249 \pm 0,117$ & $0,179 \pm 0,086$ \\
\hline Normes OMS (1995 et 2005) & 0,2 & 0,05 \\
\hline
\end{tabular}

Les résultats du tableau 3 renseignent que l'espèce Oreochromis niloticus accumule plus d'ETM que l'espèce Clarias gariepinus avec de teneurs de $0,249 \mathrm{mg} / \mathrm{kg}$ contre $0,219 \mathrm{mg} / \mathrm{kg}$ pour le $\mathrm{Pb}$; et de $0,179 \mathrm{mg} / \mathrm{kg}$ contre $0,173 \mathrm{mg} / \mathrm{kg}$ pour le $\mathrm{Cd}$. Le test de Student appliqué à ces données n'indique aucune différence significative dans les teneurs entre les deux espèces (Pb: $t=0,74 ; p=0,45 ; C d: t=0,15 ; p=0,87)$.

\section{Variation spatiale de la bioaccumulation d'Eléments Traces Métalliques (Cd et Pb)}

Les teneurs moyennes en Eléments Traces Métalliques ( $\mathrm{Pb}$ et $\mathrm{Cd}$ ) des deux espèces des poissons capturées à Baramoto et à Mongol sont présentées dans le tableau 4.

Tableau 2. Teneurs moyennes en ETM des poissons capturés sur les deux sites d'échantillonnage

\begin{tabular}{|c|c|c|c|}
\hline \multirow{2}{*}{ Sites } & \multirow{2}{*}{ Espèces } & \multicolumn{2}{|c|}{ Eléments Traces Métalliques } \\
\cline { 2 - 4 } & & $\mathbf{P b} \mathbf{( m g} / \mathbf{k g})$ & $\mathbf{C d} \mathbf{( m g} / \mathbf{k g})$ \\
\hline \multirow{2}{*}{ Baramoto } & C. gariepinus & $0,213 \pm 0,096$ & $0,195 \pm 0,082$ \\
\cline { 2 - 4 } & O. niloticus & $0,311 \pm 0,075$ & $0,173 \pm 0,067$ \\
\hline \multirow{2}{*}{ Mongol } & C. gariepinus & $0,226 \pm 0,091$ & $0,151 \pm 0,080$ \\
\cline { 2 - 4 } & O. niloticus & $0,188 \pm 0,102$ & $0,186 \pm 0,060$ \\
\hline
\end{tabular}

Les résultats du tableau 4 renseignent que chez Clarias gariepinus, la plus grande concentration moyenne en $\mathrm{Pb}$ a été enregistré à Mongole, soit $0,226 \pm 0,091 \mathrm{mg} / \mathrm{kg}$; tandis que pour le Cd, à Baramoto, soit 0,195 $\pm 0,082$ $\mathrm{mg} / \mathrm{kg}$. L’analyse statistique appliquée aux données n’a pas montré de 
différences significatives des concentrations en $\mathrm{Pb}$ et $\mathrm{Cd}$ entre les deux sites d'échantillonnages ( $\mathrm{Pb}: \mathrm{t}=-0,36 ; \mathrm{p}=0,71 ; \mathrm{Cd}$ : $\mathrm{t}=0,73 ; \mathrm{p}=0,47)$.

Chez Oreochromis niloticus, la plus grande teneur moyenne en $\mathrm{Pb}$ a été détectée à Baramoto, soit 0,311 $\pm 0,075 \mathrm{mg} / \mathrm{kg}$; en revanche la plus grande

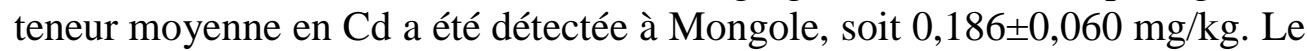
test-T appliqué aux données n'a montré aucune différence significative dans les concentrations de deux micropolluants entre les deux sites d'études $(\mathrm{Pb}: \mathrm{t}$ $=2,05 ; \mathrm{p}=0,05 ; \mathrm{Cd}: \mathrm{t}=-0,24 ; \mathrm{p}=0,81$ ).

\section{Evolution des teneurs en ETM dans les organes des poissons capturés à Baramoto}

Le tableau 5 présente les teneurs moyennes en Plomb et Cadmium détectées dans les branchies et les muscles des poissons adultes et juvéniles des espèces Clarias gariepinus et Oreochromis niloticus à Baramoto.

Tableau 5. Teneurs moyennes en ETM des poissons pêchés à Baramoto

\begin{tabular}{|l|l|l|l|l|l|}
\hline \multirow{2}{*}{ Espèces } & \multirow{3}{*}{ Stade de croissance } & \multicolumn{3}{|l|}{ Eléments Traces Métalliques } \\
\cline { 3 - 6 } & & \multicolumn{2}{|l|}{ Plomb (mg/kg) } & \multicolumn{2}{l|}{ Cadmium (mg/kg) } \\
\cline { 3 - 6 } & & Branchie & Muscle & Branchie & Muscle \\
\hline \multirow{2}{*}{ C. gariepinus } & Adulte & $0,26 \pm 0,16$ & $0,23 \pm 0,04$ & $0,27 \pm 0,13$ & $0,17 \pm 0,03$ \\
\cline { 2 - 6 } & Juvénile & $0,23 \pm 0,11$ & $0,12 \pm 0,01$ & $0,21 \pm 0,19$ & $0,1 \pm 0,02$ \\
\hline \multirow{2}{*}{ O. niloticus } & Adulte & $0,35 \pm 0,03$ & $0,3 \pm 0,02$ & $0,23 \pm 0,04$ & $0,2 \pm 0,07$ \\
\cline { 2 - 6 } & Juvénile & $0,3 \pm 0,14$ & $0,27 \pm 0,10$ & $0,14 \pm 0,06$ & $0,1 \pm 0,007$ \\
\hline
\end{tabular}

L'analyse du tableau 5 indique que, chez Clarias gariepinus, les spécimens adultes accumulent plus les deux ETM que les juvéniles, sans différence significative $(\mathrm{Pb}: \mathrm{t}=1,04 ; \mathrm{p}=0,31 ; \mathrm{Cd}: \mathrm{t}=0,55 ; \mathrm{p}=0,59)$, et cela quel que soit l'organe. La même tendance s'observe dans l'évolution des teneurs chez Oreochromis niloticus, avec une différence significative pour le $\mathrm{Cd}(\mathrm{t}=2,46 ; \mathrm{p}=0,03)$ et sans différence significative pour le $\mathrm{Pb}(\mathrm{t}=0,42 ; \mathrm{p}$ $=0,67)$.

Evolution des teneurs en ETM dans les organes des poissons capturés à Mongole

Les valeurs des teneurs moyennes en ETM observées dans les branchies et muscles des poisons adultes et juvéniles de deux espèces capturées à Mongole sont présentées dans le tableau 6.

Tableau 6. Teneurs moyennes en ETM des poissons pêchés à Mongole

\begin{tabular}{|c|c|c|c|c|c|}
\hline \multirow{3}{*}{ Espèces } & \multirow{2}{*}{$\begin{array}{c}\text { Stade de } \\
\text { croissance }\end{array}$} & \multicolumn{3}{|c|}{ Eléments Traces Métalliques } \\
\cline { 3 - 6 } & & \multicolumn{2}{|c|}{ Pb (mg/kg) } & \multicolumn{2}{c|}{ Cadmium (mg/kg) } \\
\cline { 3 - 6 } & & Branchie & Muscle & Branchie & Muscle \\
\hline \multirow{2}{*}{ C. gariepinus } & Adulte & $0,33 \pm 0,07$ & $0,21 \pm 0,01$ & $0,22 \pm 0,14$ & $0,13 \pm 0,02$ \\
\cline { 2 - 6 } & Juvénile & $0,25 \pm 0,08$ & $0,1 \pm 0$ & $0,15 \pm 0,07$ & $0,08 \pm 0$ \\
\hline O. niloticus & Adulte & $0,28 \pm 0,11$ & $0,22 \pm 0,09$ & $0,18 \pm 0,21$ & $0,15 \pm 0,05$ \\
\hline
\end{tabular}




\begin{tabular}{|l|c|c|c|c|c|}
\hline & Juvénile & $0,1 \pm 0,12$ & $0,13 \pm 0,17$ & $0,26 \pm 0,05$ & $0,13 \pm 0,07$ \\
\hline
\end{tabular}

L'examen du tableau 6 renseigne que, chez Clarias gariepinus, les teneurs moyennes en $\mathrm{Pb}$ et $\mathrm{Cd}$ sont plus élevées chez les spécimens adultes que chez les juvéniles, avec une différence significative pour le $\mathrm{Pb}(\mathrm{t}=4,58$; $\mathrm{p}=7,86)$, et sans différence significative pour le $\mathrm{Cd}(\mathrm{t}=1,00 ; \mathrm{p}=0,33)$. La même tendance a été observée chez Oreochromis niloticus, mais sans aucune différence significative dans les teneurs $(\mathrm{Pb}: \mathrm{t}=1,6 ; \mathrm{p}=0,12 ; \mathrm{Cd}: \mathrm{t}=-0,27$; $\mathrm{p}=0,79$ ).

\section{Comparaison des teneurs en ETM entre branchies et muscles des deux espèces des poissons étudiées}

La comparaison des teneurs moyennes en Eléments Traces Métalliques entre les branchies et muscles de deux espèces des poissons étudiées est reprise dans le tableau 7.

Tableau 7. Comparaison inter-organes des teneurs en ETM des poissons étudiés

\begin{tabular}{|c|c|c|c|}
\hline \multirow{2}{*}{ Espèces } & \multirow{2}{*}{ Organes } & \multicolumn{2}{|c|}{ Eléments Traces Métalliques } \\
\cline { 3 - 4 } & & $\mathbf{P b} \mathbf{( m g} / \mathbf{k g})$ & $\mathbf{C d} \mathbf{( m g} / \mathbf{k g})$ \\
\hline \multirow{2}{*}{ C. gariepinus } & Branchie & $0,272 \pm 0,075$ & $0,219 \pm 0,122$ \\
\cline { 2 - 4 } & Muscle & $0,166 \pm 0,081$ & $0,127 \pm 0,085$ \\
\hline \multirow{2}{*}{ O. niloticus } & Branchie & $0,263 \pm 0,055$ & $0,208 \pm 0,095$ \\
\cline { 2 - 4 } & Muscle & $0,236 \pm 0,082$ & $0,150 \pm 0,063$ \\
\hline
\end{tabular}

Le tableau 7 indique que, les ETM accumulés par les deux espèces sont inégalement distribués au sein de deux organes. Quel que soit l'élément étudié, les branchies concentrent plus d'ETM que les muscles, avec des teneurs de $0,272 \pm 0,075 \mathrm{mg} / \mathrm{kg}$ contre $0,166 \pm 0,081 \mathrm{mg} / \mathrm{kg}$ pour le $\mathrm{Pb}$, et $0,219 \pm 0,122$ $\mathrm{mg} / \mathrm{kg}$ contre $0,127 \pm 0,085 \mathrm{mg} / \mathrm{kg}$ pour le Cd, Chez Clarias gariepinus. De même chez Oreochromis niloticus, avec des teneurs de $0,263 \pm 0,055 \mathrm{mg} / \mathrm{kg}$ contre $0,236 \pm 0,082$ pour le $\mathrm{Pb}$, et $0,208 \pm 0,095 \mathrm{mg} / \mathrm{kg}$ contre $0,150 \pm 0,063$ $\mathrm{mg} / \mathrm{kg}$ pour le $\mathrm{Cd}$. Le test-T appliqué aux teneurs de C. gariepinus indique une différence significative pour les deux ETM $(\mathrm{Pb}: \mathrm{t}=4,9 ; \mathrm{p}=5,84$; Cd: $\mathrm{t}=3,9$; $\mathrm{p}=7,13)$; l'application du même test aux teneurs de $O$. niloticus révèle une différence significative pour le $\mathrm{Cd}(\mathrm{t}=2,74363 ; \mathrm{p}=0,01)$ et aucune différence significative pour le $\mathrm{Pb}(\mathrm{t}=0,9 ; \mathrm{p}=0,37)$.

\section{Facteur de bioconcentration des Eléments Traces Métalliques}

Les résultats des facteurs de bioconcentration d'ETM en fonction des stades de croissance de deux espèces des poissons étudiées sont présentés dans le tableau 8. 
Tableau 8. Facteur de bioconcentration en ETM des espèces de poissons suivant les sites d'études

\begin{tabular}{|c|c|c|c|c|c|}
\hline \multirow{2}{*}{ Espèces } & \multirow{2}{*}{$\begin{array}{c}\text { Stade de } \\
\text { croissance }\end{array}$} & \multicolumn{4}{|c|}{ FBC } \\
\cline { 3 - 6 } & & \multicolumn{2}{|c|}{ Baramoto } & \multicolumn{2}{c|}{ Mongole } \\
\cline { 3 - 6 } & & $\mathbf{P b}$ (mg/kg) & Cd (mg/kg) & Pb (mg/kg) & Cd (mg/kg) \\
\hline \multirow{2}{*}{$\begin{array}{c}\text { C. } \\
\text { gariepinus }\end{array}$} & Adulte & 5 & 22,8 & 27,5 & 9,1 \\
\cline { 2 - 6 } O. niloticus & Juvénile & 3,5 & 16,1 & 17,7 & 6,1 \\
\cline { 2 - 6 } & Adulte & 6,7 & 21,9 & 25,3 & 8,6 \\
\cline { 2 - 6 } & Juvénile & 5,8 & 12,6 & 12,3 & 10,1 \\
\hline
\end{tabular}

L'analyse du tableau 8 indique que les deux espèces ont bien accumulé les deux ETM présents dans leur environnement. Au niveau de Baramoto, les spécimens adultes concentrent plus les deux ETM que les juvéniles, avec les FBC de 5 pour le $\mathrm{Pb}$ et 22,8 pour le Cd chez Clarias gariepinus, et de 6,7 pour le $\mathrm{Pb}$ et 21,9 pour le Cd chez Oreochromis niloticus. La même situation s'observe à Mongole chez C. gariepinus (27,5 pour le $\mathrm{Pb}$ et 9,1 pour le $\mathrm{Cd}$ ), tandis que chez $O$. niloticus, les juvéniles accumulent plus le Cd $(10,1)$ que les adultes, qui sont les plus fort concentrateurs de $\mathrm{Pb}(25,3)$.

\section{Discussion}

Les résultats des analyses physico-chimiques des sites de prélèvement des poissons ont montré que les eaux ont un $\mathrm{pH}$ légèrement acide, avec une température moyenne allant de $26,4 \pm 1,27^{\circ} \mathrm{C}$ à Baramoto et $27,3 \pm 0,79{ }^{\circ} \mathrm{C}$ à Mongole, et une conductivité faible. Ces paramètres mesurés sont comprises dans les limites préconisées par l'OMS (2011) soit $(6,5$ - 8,5) pour le $\mathrm{pH}$ et $\left(25-29^{\circ} \mathrm{C}\right)$ pour la température contrairement à la conductivité qui est restée inférieure aux mêmes normes $(50-2000 \mu \mathrm{S} / \mathrm{cm})$. Ces observations sur la qualité physico-chimique des eaux du Pool Malebo corroborent à celles de Pwema (2014), qui a signalé une conductivité faible entre 28,9 et 52,4 $\mu \mathrm{S} / \mathrm{cm}$, un $\mathrm{pH}$ légèrement acide oscillant autour de 6,1 à 6,3, avec une température moyenne de l'eau variant entre 27,6 et $28,3{ }^{\circ} \mathrm{C}$. Tembeni (2017) souligne également un $\mathrm{pH}$ légèrement acide avec des valeurs oscillant autour de 6,3 à 6,55 , avec une température moyenne de l'eau de $26,4^{\circ} \mathrm{C}$, et une conductivité de 35,27 à 43,53 $\mu \mathrm{S} / \mathrm{cm}$.

La présente étude a mis en évidence la présence de $\mathrm{Cd}$ et $\mathrm{Pb}$ dans tous les compartiments retenus (eau-sédiment-poissons) à la station de pêche de Kingabwa. Les teneurs en Eléments Traces Métalliques mesurés dans les eaux provenant de deux sites ont varié entre 0,01 et $0,02 \mathrm{mg} / \mathrm{L}$ pour le $\mathrm{Cd}$ et 0,01 à $0,05 \mathrm{mg} / \mathrm{L}$ pour le $\mathrm{Pb}$. Ces teneurs se sont révélées supérieures au seuil recommandé par l'OMS (2017) qui est de $0,003 \mathrm{mg} / \mathrm{L}$ pour le Cd et de $0,01 \mathrm{mg} / \mathrm{L}$ pour le $\mathrm{Pb}$. Par contre, la teneur en $\mathrm{Pb}$ dans les eaux prélevées à Mongole a été à la limite de la norme (soit $0,01 \mathrm{mg} / \mathrm{L}$ ). Au regard de la norme précitée, cette situation indique qu'il pourrait y avoir pollution en ETM dans 
ce compartiment. Cette observation est en harmonie à celle de Nzapo et al., (2018) qui ont révélé des teneurs supérieures $(\mathrm{Cd}: 0,12$ à $0,13 \mathrm{mg} / \mathrm{L} ; \mathrm{Pb}: 0,12$ à $0,13 \mathrm{mg} / \mathrm{L}$ ) dans les eaux du Pool Malebo à Kingabwa par rapport aux teneurs déterminées dans la présente étude. Ceci pourrait s'expliquer par les méthodes utilisées pour la détection des Eléments Traces Métalliques; la technique spectrophotométrique d'absorption atomique, tandis que la présente étude a utilisé la méthode d'analyse spectrophotométrique d'absorption moléculaire (Uv-visible).

Quant aux sédiments, les valeurs obtenues sur les deux sites sont restées en générale plus faibles, fluctuant entre 0,01 à $0,04 \mu \mathrm{g} / \mathrm{g}$ pour le $\mathrm{Cd}$, et 0,01 à $0,02 \mu \mathrm{g} / \mathrm{g}$ pour le $\mathrm{Pb}$. Ces valeurs sont en dessous de la limite de recommandation du CCME (Cd: $600 \mu \mathrm{g} / \mathrm{g}$; Pb: $35000 \mu \mathrm{g} / \mathrm{g}$. Les teneurs détecter dans ce compartiment sont inférieures à celles obtenues par Mata et al., (2020) pour le Cd (0,02 à 6,65 mg/kg) et pour le $\mathrm{Pb}(2,36$ à 200,89 mg/kg) dans les sédiments de surface de sept stations du Pool Malebo. Mwanamoki et al., (2014) dans une étude approfondie sur la distribution d'Eléments Traces dans les sédiments du Pool Malebo, ont mis en évidence des teneurs supérieures à celles de la présente étude. La différence avec ces auteurs serait due aux périodes d'échantillonnages des études.

Les valeurs des ETM ( $\mathrm{Cd}$ et $\mathrm{Pb}$ ) enregistrées dans les eaux et les sédiments de Kingabwa indiquent que l'eau est plus chargée en Eléments Traces Métalliques que les sédiments. Cette situation peut s'expliquer par les conditions physico-chimiques du milieu, notamment le $\mathrm{pH}$. En effet, le $\mathrm{pH}$ acide favorise la biodisponibilité d'ETM dans la solution ainsi que leur mobilité le long du cours d'eau. Par contre, lorsqu'il est basique, les ETM précipitent et s'accumulent dans les sédiments, accroissant ainsi leurs concentrations (Kashimbo et al., 2016). Les valeurs obtenues dans cette étude pour l'eau, sont également inférieures à celles rapportées par Muteba et al., (2011) (Cd: 1,42 mg/L; Pb: 0,24 mg/L) dans les eaux de la rivière Kafubu et à celles de Katemo et al., (2010) (Cd: 2,838 mg/L; Pb: 0,011 mg/L) dans le bassin de la Lufira, dans le Haut-Katanga en R.D Congo. Cette différence des teneurs avec ces auteurs peut s'expliquer du fait que le Haut-Katanga est une zone à haute exploitation minière, et les écosystèmes de cette zone seraient plus exposés aux ETM que notre zone d'étude.

Les résultats d'analyses toxicologiques des poissons de la station de Kingabwa ont révélés la présence de $\mathrm{Cd}$ et $\mathrm{Pb}$ à des concentrations assez importantes; ces concentrations oscillent entre 0,173 et $0,179 \mathrm{mg} / \mathrm{kg}$ pour le $\mathrm{Cd}$, et entre 0,219 et $0,249 \mathrm{mg} / \mathrm{kg}$ pour le $\mathrm{Pb}$. En se référant aux seuils de recommandations fixés par l'OMS (1995 et 2005) sur la comestibilité des poissons ( $\mathrm{Pb}: 0,2 \mathrm{mg} / \mathrm{kg}$; $\mathrm{Cd}: 0,05 \mathrm{mg} / \mathrm{kg}$ ), nos résultats montrent que les deux espèces de poissons étudiées sont contaminées par les ETM. De ce fait, 
elles pourraient donc engendrées des problèmes pour la santé humaine au fil du temps.

La comparaison des teneurs en Eléments Traces Métalliques dans les poissons aux différents sites étudiés, montre que les espèces capturées à Baramoto renferment des concentrations supérieures par rapport à celles de Mongole, mais cela sans différence significative. Ceci peut s'expliquer du fait de la fréquence élevée d'activités anthropiques observées sur ce site (décharges industrielles, rejets domestique, rejets accidentelles de carburant et huile de moteur par les transporteurs des barges qui remontent le fleuve Congo, vidange des fosses septiques).

Les résultats d'analyses faites par Nsimanda et al., (2015a et b); Nzapo et al., (2018) corroborent avec les notre dans la mesure où ils confirment la présence des Eléments Traces Métalliques chez les poissons du Pool Malebo. Comparativement à ces travaux, la présente étude a révélée des teneurs en ETM inferieures à celles rapportées par Nsimanda et al., (2015a) chez Distichodus fasciolatus ( $\mathrm{Pb}: 12,48 \mathrm{mg} / \mathrm{kg}$; Cd : 8,34 mg/kg), Mormyrops anguilloïdes ( $\mathrm{Pb}: 28,91 \mathrm{mg} / \mathrm{kg}$; Cd : 9,44 mg/kg) et Schilbe mystus (Pb: 75,22 $\mathrm{mg} / \mathrm{kg}$; Cd: 15,29 mg/kg) à la station de Kinsuka. Ceci s'explique par le fait que la station de Kinsuka est le carrefour de concentrations des tous les polluants de la ville (rejet industriel et domestique, ruissellement, etc.). Par contre, nos résultats restent supérieurs à ceux des mêmes auteurs chez $D$. fasciolatus ( $\mathrm{Pb}: 0,003 \mathrm{mg} / \mathrm{kg}$; Cd: 0,001 mg/kg), M. anguilloïdes ( $\mathrm{Pb}: 0,005$ $\mathrm{mg} / \mathrm{kg}$; Cd : 0,001 mg/kg) et $S$. mystus (Pb: 0,007 mg/kg; Cd : 0,002 mg/kg) à la station de pêche de Maluku. Cette différence des teneurs en ETM à ce niveau serait due au fait que les espèces de poissons étudiées se diffèrent. Mais aussi, la faible fréquence d'activités anthropiques observées sur la station de pêche de Maluku, une zone avec moins d'activités humaines que la zone de Kingabwa pouvait aussi influencer l'accumulation des ETM chez les poissons. Le même constat fait chez les poissons de la station de Kingabwa a été aussi relevé par Nzapo et al., (2018).

Quant à la variation des concentrations en Eléments Traces Métalliques dans les organes de Clarias gariepinus et Oreochromis niloticus, il a été constaté que les branchies accumulent plus d'ETM que les muscles. Ces fortes concentrations observées dans les branchies se justifient par le fait que cet organe intervient directement dans la filtration de l'eau au contact du poisson. La même observation a été faite par Chadid (2016) chez Sardina pilchardus. En outre, Drevet (2014) révèle que les branchies contiennent un taux élevé en métallothionéine par rapport aux muscles, qui intervient dans la séquestration d'ETM.

Les valeurs du FBC enregistrées chez les deux espèces de poissons varient entre 3,5 à 27,5 pour le $\mathrm{Pb}$, et de 6,1 à 22,8 pour le $\mathrm{Cd}$. La variation de ces valeurs montre que les espèces du site de Baramoto accumulent plus le Cd, 
tandis que celles de Mongole accumulent plus le $\mathrm{Pb}$. Ceci serait probablement lié à la fraction assimilable présente dans chaque site. Nos résultats du FBC ont révélé des valeurs inférieures à celles obtenues par Diop et al., (2019) qui ont rapporté des valeurs variant entre 44 et 506 pour le $\mathrm{Pb}$, et entre 8 et 65 pour le Cd chez les espèces de Tilapia, dans cinq étangs de Dakar. La différences des résultats obtenus avec ceux de cet auteur serait lié à la différence des écosystèmes aquatiques exploités et du temps de résidence des eaux (Blanquet et al., 2004). La présente étude a été réalisée dans un milieu lotique, contrairement à cette dernière, dans un milieu lentique. D'une manière générale, la mesure de l'intensité de la bioaccumulation suivant le stade de croissance a montré que les poissons adultes accumulent plus les deux ETM que les juvéniles. Ceci pourrait s'expliquer par le temps d'imprégnation qui augmente avec l'âge (taille) et qui a un effet sur l'accumulation d'ETM lorsque les concentrations des métaux sont très élevées dans l'eau (Ennouri et al., 2013).

La comparaison interspécifique de la variation de concentrations de deux ETM (Pb et $\mathrm{Cd}$ ) chez les deux espèces de poissons étudiées a montré que Oreochromis niloticus accumule davantage les ETM que Clarias gariepinus, mais cela sans différence significative. Nous avons constaté, au travers de cette variation, que les espèces pélagiques pourront être plus vulnérables à la contamination aux Eléments Traces Métalliques que les espèces benthiques. Les travaux réalisés par Kidd et al., (2003) ; Power et al., (2002) ont montré que les espèces pélagiques accumulent plus d'ETM que les espèces benthiques. Toutefois, d'après Casas (2005), le concept de la bioaccumulation résulte de plusieurs mécanismes agissant simultanément ou avec un décalage dans le temps. La bioaccumulation, pour un même composé chimique, peut varier considérablement selon l'espèce considérée, le stade de développement des individus, les variables environnementale $(\mathrm{pH}$, salinité et température, etc.), le sexe, le régime alimentaire, les propriétés du contaminant, etc.

\section{Conclusion Et Suggestions}

Cette étude a montré que la station de Kingabwa dans le Pool Malebo (fleuve Congo) est bel et bien contaminée par le Cadmium et le Plomb. Les eaux des différents sites d'échantillonnage, ont présentées des teneurs en ETM supérieures aux seuils recommandés par l'OMS pour l'eau potable. Les résultats d'analyse des sédiments ont révélés des faibles concentrations par rapport aux normes édictées par le CCME pour les sédiments d'eau douce. Les concentrations d'ETM trouvées dans les organes de deux espèces des poissons étudiées ont été généralement au-dessus de normes préconisées par l’OMS pour la consommation; de ce fait elles constituent un risque pour la santé de la population consommatrice. Il a été démontré au cours de cette étude que, les eaux renferment plus d’ETM que les sédiments alors que le poisson pélagique, 
Oreochromis niloticus est plus contaminé en $\mathrm{Cd}$ et $\mathrm{Pb}$ que le poisson benthique, Clarias gariepinus. Nous conseillons à la population riveraine du fleuve Congo dans le Pool Malebo d'éviter une consommation régulière des poissons qui proviennent des zones à fortes concentrations en ETM mais aussi, d'éviter l'utilisation des eaux du Pool Malebo dans les processus domestiques.

\section{Remerciements}

Les auteurs remercient les pêcheurs du Pool Malebo en général dont ceux de Kingabwa pour leur apport durant la collecte des données ainsi que les responsables du Laboratoire de Géochimie du Centre de Recherches Géologiques et Minières de Kinshasa pour les analyses des échantillons.

\section{References:}

1. Blanquet, J.P., Le Goff, F., Coquery, M., Gaudillot, A., Gondelle, F., Houeix, N., Magaud, H., Meunier, L., Sanchez, W. \& Vincent B. (2004). Devenir et comportement des métaux dans l'eau : biodisponibilité et modèles BLM. Rapport technique, Institut National de la Recherche Scientifique, Québec, Canada, 85 p.

2. Casas, S. (2005). Modélisation de la bioaccumulation de métaux traces ( $\mathrm{Hg}, \mathrm{Cd}, \mathrm{Pb}, \mathrm{Cu}$ et $\mathrm{Zn}$ ) chez la moule, Mytilus galloprovincialis en milieu méditerranéen. Thèse de Doctorat, Université du Sud Toulon. France, $301 \mathrm{p}$.

3. CCME disponible sur: http://www.ccme.ca/recommandationcanadienne-pour-la-qualité-de-l'environnement. Consulté le 09/01/2020.

4. Chadid, A. (2016). Quantification des éléments traces métalliques (cadmium, plomb et mercure total) de certains produits de la pêche débarqués dans la zone Essaouira-Dakhla : Evaluation des risques sanitaires. Thèse de Doctorat, Université Ibn Zohr. Maroc, 191 p.

5. Diop, C., Diatta, A., Ndiaye, A., Cabral, M., Toure, A. \& Fall, M. (2019). Teneurs en métaux traces des eaux et poissons de cinq étangs de Dakar et risques pour la santé humaine. Journal of Applied Biosciences, 137 : 13931-13939.

6. Drevet, O. (2014). Exposition de la perchaude (Perca flavescens) à un mélange de cadmium et nickel: répartition subcellulaire des métaux accumulés et conséquences métaboliques. Mémoire de Master, Université du Québec, Institut National de la Recherche Scientifique Centre Eau Terre Environnement, Canada, 82 p.

7. Ennouri, R., Mili, S. \& Chouba, L. (2013). La contamination métallique du rouget de vase (Mullus barbatus) et de la sardinelle (Sardinella aurita) du golfe de Tunis. Cybium, 37(1-2): 49-58. 
8. Falasi, N. (2018). Pollution de la rivière N'djili et contraintes de gestion des sols autour du Pool Malebo (cas du site agricole Masina Rail 1/Kinshasa). Mémoire de DEA, Université de Liège, Belgique, 64 p.

9. Foxall, C., Chale, F., Bailey-Watts, A., Patterson, G. \& West, K. (2000). Les pesticides et les métaux lourds dans les poissons et les mollusques du Lac Tanganyika. Lake Tanganyika Biodiversity Project, République Démocratique du Congo, 16 p.

10. Kashimbo, K. S., Mukanya, S. S., Mukoj, K. A., Mwenge T. L., Kesonga, N. M., Meli, K. \& Kiyukeno, K. Y. (2016). Etudes d'impacts des rejets liquides de l'usine STL (Société de Terril de Lubumbashi) sur la rivière Lubumbashi (Haut-Katanga/RD. Congo). International Journal of Innovation and Scientifique, Research, 21(2) : 285-292.

11. Katemo, B. M., Colinet, G., André, L., Chocha, A. M., Marquet, J- P. \& Micha, J- C. (2010). Evaluation de la contamination de la chaine trophique par les éléments traces $(\mathrm{Cu}, \mathrm{Co}, \mathrm{Zn}, \mathrm{Pb}, \mathrm{Cd}, \mathrm{U}, \mathrm{V}$ et $\mathrm{As})$ dans le bassin de la Lufira supérieure (Katanga/RD Congo). Tropicultura, 28(4) :246-252.

12. Kidd, K. A., Bootsma, H. A., Hesslein, R. H., Lockhart, L. \& Hecky, R. E. (2003). Mercury concentrations in the food web of Lake Malawi, East Africa. Journal of Great Lakes Research, 29 :258-266.

13. Laprieur, F. \& Rubin, A. (2011). INVAQUA : Prédiction de l'établissement des espèces exotiques dans les milieux aquatiques : vers une anticipation des invasions biologiques. Rapport scientifique. France, $131 \mathrm{p}$.

14. Lévêque, C., Paugy, D. \& Teugels, G. G. (1990). Faune des poissons d'eaux douces et saumâtres d'Afrique de l'Ouest. Tome 1, MRAC, O.R.S.T.O.M, Collection faune tropicale, $\mathrm{n}^{\circ} 28,902 \mathrm{p}$.

15. Lévêque, C., Paugy, D. \& Teugels, G. G. (1992). Faune des poissons d'eaux douces et saumâtres d'Afrique de l'Ouest. Tome 2, MRAC, O.R.S.T.O.M, Collection faune tropicale, $\mathrm{n}^{\circ} 28,902 \mathrm{p}$.

16. Mata, K. H., Mohammed, M. A. D., Ngweme, N. G., Konde, N. J., Mulaji, K. C., Kiyombo, M. G. \& Poté, W. J. (2020). Toxique metal concentration and ecotoxicity test of sediments from dense populated areas of Congo River, Kinshasa, Democratic republic of the Congo. Environemental chemistry and ecotoxicology, 2 : 83-90.

17. Mbega, J. D. \& Teugels, G. G. (2003). Guide de détermination des poissons du Bassin inférieur de l'Ogooué. Presse Universitaire de Namur, Belgique, 165 p.

18. Monama, O., Mukinayi, J. \& Sibukaku, S. (1985). Chaine trophique du plomb. Revue Zaïroise des Sciences Nucléaires, 6 :226-237. 
19. Muteba, L. J- P., Kitoko, N. F., Moma, T. M. B. \& Mande, S. M. (2011). Pollution de la rivière Kafubu. Rapport d'enquête, 97 p.

20. Mwanamoki, P. M., Naresh, D., Niane, B., Ngelinkoto, P., Thevenon, F.,. Nlandu, J. W., Mpiana, T. P., Prabakar, K., Mubedi, I. J., Kabele, G. C., Wildi, W. \& Poté, J. (2014). Trace metal distributions in the sediments from river-reservoir systems: case of the Congo River and Lake Ma Vallée, Kinshasa (Democratic Republic of Congo. Environ Sci. Pollut Res, $10: 11356-3381$.

21. Nsimanda, I. C., Musibono, E. D., Basosila, L. N. \& Wanga, B. M. B. (2015a). Etude préliminaire de la contamination au Cadmium et au Plomb de Distichodus fasciolatus, Mormyrops anguilloides et Schilbe mystus au Pool Malebo (fleuve Congo - Kinshasa/R.D Congo) à Kinsuka. International Journal of Innovation and Applied Studies, 10(1) :176-183.

22. Nsimanda, I. C., Musibono, E. D., Basosila, L. N. \& Wanga, B. M. B. (2015b). Contribution à l'étude écotoxicologique du Cadmium et du Plomb dans Distichodus fasciolatus, Mormyrops anguilloides et Schilbe mystus au Pool Malebo (fleuve Congo - Kinshasa/R.D Congo) à Maluku. International Journal of Innovation and Applied Studies, 10(1) : 166-175.

23. Nzapo, K. H, Ngbolua, Koto-te-Nyiwa., Bongema A. L., Bongo N. G., Inkoto L. C, Falanga M. C., Ashande M. C., Ndembo N. J. L., Lokilo L. E. \& Djoza D. R. (2018). Evaluation de la bioaccumulation de métaux lourds chez Clarias gariepinus (Burchell, 1822), Chrysichthys nigrodigitatus (Lacepède, 1803), Mormyrops anguilloides (Linnaeus, 1758) et Coptodon rendalli (Boulenger, 1897). International Journal of Innovation and Scientific Research, 38(1) : 185-191.

24. OMS. (1995). Normes générale codex pour les contaminants et les toxines présents dans les produits de la consommation humaine et animale. Codex standard 193-1995, 43 p.

25. OMS. (2005). Liste provisoire des principales espèces de poissons faisant l'objet d'un commerce internationales (y compris les propositions concernant des concentrations maximales de plomb dans différentes espèces de poissons). Trente septièmes sessions, La Haye, Pays-Bas, 4 p.

26. OMS. (2017). Directives de qualité pour l'eau de boisson. 4è Edition, Genève, Suisse, $539 \mathrm{p}$.

27. Ouédraogo, A. (2016). Évaluation de la teneur en métaux lourds, dans les tissus du Tilapia du Nil (Oreochromis niloticus) et du poisson chat africain (Clarias sp) d'eau douce du Burkina-Faso. Mémoire de Licence, Université Polytechnique de Bobo- Dioulasso, Burkina-faso, $73 \mathrm{p}$. 
28. Poll, M. \& Gosse J. P. (1995). Genera des poissons d'eau douce de l'Afrique. Classe des Sciences, Académie Royale de Belgique. 324 p.

29. Power, M., Klein, G. M., Guiguere, K. R. R. A. \& Kwan, M. H. (2002). Mercury accumulation in the fish community of a sub-artic lake in relation to trophic position and carbon sources. Journal of Applied Ecology, 39 : 819-830.

30. Pwema, K. V. (2014). Ecologie alimentaire, reproduction et modes d'adaptation de cinq espèces du genre Labeo Cuvier, 1817 dans les milieux lentiques et des rapides au Pool Malebo dans le fleuve Congo. Thèse de Doctorat en Sciences Biologiques, Université de Kinshasa. RD. Congo, $163 \mathrm{p}$.

31. Pwema, K. V., Mbomba, B. N., Kikala, A. E., Lusasi, S. W. \& Micha, J- C. (2019). Utilisation des alevins de Schilbe mystus (Linné, 1758) (Siluriformes : Schilbeidae) dans la lutte biologique contre les larves de moustiques. Congo science, 7(1) : 83-86.

32. Tembeni, M. T. J. (2017). Structure des communautés des poissons Mochokidae (Ostariophysi, Siluriformes) dans le Pool Malebo (Fleuve Congo/R.D. Congo) en relation avec la distribution spatio-temporelle, la niche trophique et la stratégie démographique. Thèse de Doctorat en Sciences Biologiques, Université de Kinshasa, R.D. Congo, 303 p.

33. WHO (World Health Organization). (2011). Guidlines for drinkingwater quality. Fourth édition, Genève, Suisse, 564 p. 